\title{
Hepatoprotective efficiency of selenolin studied on the model of acute liver failure in rats
}

\author{
Marina P. Semenenko*, Vladimir A. Grin, Ksenia A. Semenenko, Elena V. Kuzminova, and \\ Eugenia $V$. Rogaleva \\ Krasnodar Research Center for Animal Husbandry and Veterinary Medicine, 350055 Krasnodar, \\ Russian Federation
}

\begin{abstract}
The influence of selenolin on the metabolic functions of the liver of laboratory rats was studied on the model of the reproduction of acute liver failure caused by acetaminophen. It was determined that the use of selenolin inhibits and weakens the hepatotoxic effects, contributing to the correction of impaired homeostasis in rats. Under the action of the preparation, there was a decrease in enzymatic activity - AST - in 2.35 times, ALT - in 2.4 times, $\gamma$-GGT - by $21.7 \%$, as well as an improvement in protein synthesizing function, manifested by an increase in the concentration of total protein by $26.9 \%$. Selenolin significantly $(p<0.05)$ reduces the level of lipid peroxidation products - DC - in 1.5 times, CD by $56.3 \%$, MDA - by $19.9 \%$. Due to the organic selenium included in the preparation, selenolin helps to maintain a high activity of the enzyme antioxidant system, which leads to a decrease in the stationary level of free radical and lipid peroxidation products, allowing the body to quickly cope with pathological changes in the liver cells and in the blood homeostasis system.
\end{abstract}

\section{Introduction}

The purpose of studying the specific activity of drugs is to conduct pathophysiological experiments that allow reproducing models of acute and chronic diseases in laboratory animals, and then, using these models, to identify the patterns of their development, starting from the moment the etiological factor (toxicant) interacts with the body until the outcome. Using a model experiment, it is possible to determine the relationship between damage and the functional state of various organs, systems and the body as a whole $[1,2]$.

The means of metabolic therapy of liver diseases include all substances that have hepatoprotective activity, including the pronounced antioxidant effect and potentiation of endogenous antioxidant systems of hepatocytes, inhibition of phospholipolysis with a decrease in the production of lysophosphatides and restoration of the normal spectrum of membrane phospholipids, deposition of calcium ions, as well as improvement matrix and barrier functions of cytolemma, mitochondrial membranes, endoplasmic reticulum (EPR) and lysosomes $[3,4]$.

*Corresponding author: sever291@mail.ru 
In the mechanisms of development of liver diseases, one of the essential features is the activation of lipid peroxidation (LPO) processes, leading to damage of hepatocytes at the membrane level [5]. Excessive formation of free radicals contributes to a change in the physicochemical state of the lipid matrix, a decrease in the hydrophobicity of the lipid layer of membranes, and an increase in their permeability. Under conditions of the liver pathology, the intensity of LPO depends on many factors, correlating both with the activity of the pathological process and with the functional state of the antioxidant system of hepatocytes. The main role in the destruction of unsaturated fatty acid hydroperoxides formed during LPO plays the glutathione system, in particular, glutathione peroxidase glutathione reductase.

Therefore, it becomes clear that researchers are interested in the antioxidant properties of selenium, which is part of the active center of glutathione peroxidase, and its ability to have a hepatoprotective effect.

The aim of the research was to clarify the degree and nature of violations of blood homeostasis and free radical oxidation of lipids, as well as the selenium status in the dynamics of the development of acute liver failure in laboratory animals.

\section{Materials and methods}

Evaluation of the hepatoprotective properties of selenolin was studied on laboratory white nonlinear rats during the reproduction of acute liver failure - a clinical and laboratory syndrome that occurs in response to severe damage of liver cells, characterized by severe disruption of the functional activity of hepatocytes and manifested by the rapid increase in intoxication in the animal body.

Acetaminophen (paracetamol), the most common representative of the group of nonsteroidal anti-inflammatory drugs, was used as a hepatotoxic agent. The hepatotropicity of the drug is associated with its metabolism in the liver. Metabolism of paracetamol at therapeutic dosages is carried out by glucuronidation and sulfonation, and the resulting products are excreted in the urine. When using high doses of the drug, these mechanisms are saturated and paracetamol begins to metabolize in the cytochrome P-450 system with the formation of a toxic metabolite $-\mathrm{N}$-acetyl-p-benzoquinone imine (NAPQI), which has direct damaging effects on hepatocytes: disturbance of calcium flow in mitochondria, formation of hydroxyl radicals, formation of nitrites and nitrates that leads to the activation of apoptosis.

For the experiment, four groups of adult white rats $(n=8)$ with an average body weight of 180-215 g were formed. Animals of the first two groups (experimental) were injected intramuscularly with selenolin at doses of $0.2 \mathrm{ml} /$ animal and $0.4 \mathrm{ml} /$ animal, and then at the same dosages one hour before poisoning and then every 7 days of the experimental period (within three weeks). The animals of the third group (control) were injected with saline at a dose of $0.4 \mathrm{ml} /$ animal according to the similar scheme.

In addition, in the design of the experiment, healthy rats (intact control) that were not subjected to poisoning were used, from which at the final stage of the study blood was taken for a comparative assessment of the biochemical parameters of healthy animals and animals subjected to poisoning.

The calculation of the dose of acetaminophen (paracetamol) was carried out on the basis of dividing the maximum daily dose for a person $(4000 \mathrm{mg})$ by the weight of an adult $(60$ $\mathrm{kg}$ ) and multiplying the final result by 7 (coefficient for a laboratory rat), which was 470 $\mathrm{mg} / \mathrm{kg}$ of body weight. Propylene glycol was chosen as the solvent.

A working solution of paracetamol was prepared by adding dry powder to an $80 \%$ (vol.) of propylene glycol solution in distilled water, followed by stirring and heating the composition to $40{ }^{\circ} \mathrm{C}$. The resulting solution was stable after cooling, its homogeneity was 
preserved, no precipitation occurred. The administration of the drug solution to rats was carried out intraperitoneally in a volume of $2 \mathrm{ml}$ using a standard sterile syringe with a capacity of $5 \mathrm{~cm}^{3}$.

The hepatoprotective effect of selenolin was evaluated within the framework of drug correction of acute hepatic failure according to the safety of animals, clinical condition, biochemical homeostasis with an emphasis on the hepatological profile, indicators of antioxidant activity and histological examination of the liver.

\section{Results and discussion}

The development of acute liver failure in animals was accompanied by the following clinical manifestations: progressive depression, convulsions, ataxia (impaired coordination), increased body temperature, rapid breathing, cyanosis, in some cases, diarrhea with increasing dehydration, increased thirst. With experimental toxic liver damage in laboratory animals, starting from the 7th day, acute liver intoxication, as a rule, is already transformed into a chronic one, therefore, on the following days of observations, some of the experimental rats began to show symptoms of cholestasis (yellowness of the mucous membranes and skin, itching).

The clinical signs of the modeled pathology persisted for 8-10 days, after which their intensity began to weaken. At the same time, in the third experimental group on the 6th and 9th days of the experimental period, the death of two animals was recorded. Posthumous autopsy of dead rats revealed signs of acute damage of the liver tissue, which was characterized by the presence of dystrophic and necrotic areas of the parenchyma, localized mainly in the central part of the organ. In addition, changes in the stomach and intestines caused by hemorrhages and multiple hemorrhages of the mucous membrane were noted in both animals. There were no pathological changes in other organs.

Histological examination in most of the micro preparations revealed circulatory disorders of the hepatic lobules in the form of pronounced plethora of central veins, sinusoidal capillaries (mainly in the central part), as well as signs of dystrophic changes in hepatocytes.

In a number of cases, areas of necrotic damage of liver cells were identified, accompanied by cell lysis.

The results of the biochemical study of the blood serum of animals showed that the administration of selenolin on the background of drug-induced acute liver failure contributed to the correction of the impaired homeostasis of rats (Table 1).

Table 1. Biochemical parameters of blood serum in model liver failure with acetaminophen (paracetamol), $\mathrm{M} \pm \mathrm{m}, \mathrm{n}=5$

\begin{tabular}{|l|c|c|c|c|}
\hline \multirow{2}{*}{ Indicators } & \multicolumn{4}{|c|}{ Groups of animals } \\
\cline { 2 - 5 } & Experimental 1 & Experimental 2 & Control & Intact \\
\hline AST, AU/ 1 & $96.5 \pm 3.11$ & $74.7 \pm 2.87^{*}$ & $175.3 \pm 1.64$ & $38.4 \pm 3.28$ \\
\hline $\mathrm{ALT}, \mathrm{AU} / \mathrm{l}$ & $63.2 \pm 4.02$ & $51.9 \pm 3.56$ & $124.7 \pm 4.71$ & $32.1 \pm 2.50$ \\
\hline $\mathrm{ALP}, \mathrm{AU} / \mathrm{l}$ & $357.4 \pm 11.3^{* *}$ & $328.8 \pm 9.72$ & $542.0 \pm 6.84$ & $286.3 \pm 5.47$ \\
\hline$\gamma$-GGT, nanomol / & $8.7 \pm 0.86$ & $8.4 \pm 0.71^{* *}$ & $15.6 \pm 1.23$ & $6.81 \pm 0.58$ \\
\hline Total $\mathrm{protein} \mathrm{g} / \mathrm{l}$ & $56.7 \pm 1.14$ & $63.1 \pm 1.88$ & $49.7 \pm 2.53$ & $70.6 \pm 3.41$ \\
\hline Urea, $\mathrm{mmol} / \mathrm{l}$ & $4.16 \pm 0.53$ & $5.27 \pm 0.27$ & $5.69 \pm 0.76$ & $3.85 \pm 0.48$ \\
\hline Cholesterol $\mathrm{mmol} / 1$ & $2.1 \pm 0.21$ & $2.2 \pm 0.19$ & $3.5 \pm 0.53$ & $1.9 \pm 0.09$ \\
\hline Total bilirubin $\mu \mathrm{mol} / 1$ & $9.7 \pm 1.07^{*}$ & $9.4 \pm 0.98^{* *}$ & $11.8 \pm 1.14$ & $8.2 \pm 0.56$ \\
\hline Creatinine, $\mu \mathrm{mol} / 1$ & $36.5 \pm 4.22$ & $40.2 \pm 2.17^{*}$ & $53.9 \pm 3.67$ & $28.7 \pm 1.27$ \\
\hline
\end{tabular}

Note: - the differences are significant $* \mathrm{p}<0.05 ;{ }^{* *} \mathrm{p}<0.01$ in comparison with the negative control group 
Table data demonstrate the positive effects of selenolin on a number of blood biochemical parameters. Preventive administration of the drug before the development of acute liver failure in laboratory animals contributed to a decrease in the enzymatic activity of liver cells relative to the negative control group. At the same time, the differences in aspartate aminotransferase between the experimental and control groups were 1.86 and 2.35 times in favor of the rats that received drug correction. A similar difference was recorded in the activity of alanine aminotransferase -1.97 and 2.4 times against the indicators of the control group. It should be noted that the values of transaminases in the experimental groups at the end of the experiment were still quite high, as indicated by the differences with the group of intact animals. In comparison with them, the level of AST in the first experimental group exceeded similar values in healthy analogues in 2.5 times, ALT - in 1.97 times. The same picture was observed in the activity of transaminases of the second experimental group - the increase was in 1.94 and 1.61 times. It is known that liver cells are capable of complete regeneration after being damaged by toxic agents. Moreover, the regenerative function of the liver is stimulated by necrosis of hepatocytes, and if the connective tissue frame of the organ remains intact, then it is quite probable that the structure of the liver is completely restored. In our case, with liver damage, there was a massive damage not only to the membranes of hepatocytes, causing the release of a cytoplasmic enzyme into the blood, which is ALT, but also the sequential destruction of the cellular structure itself, including cellular organelles - mitochondria, which led to an increase in the degree of AST. However, the dynamic decrease in transamination enzymes in the groups with drug correction indicates an increase in the degree of liver regeneration in the experimental rats and its subsequent recovery. Moreover, the dose of selenolin, which was $0.2 \mathrm{ml} /$ animal, turned out to be less effective than the dose of $0.4 \mathrm{ml} /$ animal, which, to a greater extent, contributed to a decrease in cytolysis of hepatocytes.

In the negative control group, the activity of enzymes (all of them) not only did not decrease, but remained significantly high even three weeks after inoculation, which may indicate the development and subsequent preservation of signs of liver failure. At the same time, the value of the $\mathrm{R}$ index (the ratio of the level of alanine transaminase to the level of alkaline phosphatase) in animals was 0.22 , which suggests hepatocellular liver damage, despite the fact that the level of alkaline phosphatase in the blood of rats was quite high even three weeks after inoculation with paracetamol. The differences with the indices of the experimental groups were 1.52 and 1.65 times higher, respectively. The difference with intact animals was 1.89 times higher.

When assessing $\gamma$-GGT (gamma-glutamyltransferase), its moderate increase was revealed in all groups of animals exposed to drug intoxication. However, in the groups with the use of selenolin, the increase in this value by the end of the experimental period was less pronounced (the difference with the group of healthy animals was $21.7 \%$ and $18.9 \%$ ). Whereas in the blood of control rats the level of $\gamma$-GGT exceeded the value of this enzyme in relation to the indices of intact rats in 2.3 times. The intergroup differences with the rats receiving selenolin in the control animals were in 1.8 and in 1.86 times. And if we consider other indicators of cholestasis - alkaline phosphatase, cholesterol and total bilirubin, we can conclude that on the background of acute liver inflammation in rats of the control group, signs of cholestasis development began to appear, while in experimental rats of the first and second groups the use of selenolin allowed to stop the development of the acute process and prevent the disease from going into the chronic stage to a certain extent.

This is also indicated by parameters of protein metabolism, in particular, the level of total protein. By the end of the study, the concentration of total protein in the experimental groups increased by $14 \%$ and $26.9 \%$. Moreover, in the second group, this increase reached the lower boundaries of the species norm. In the negative control group, the values of total 
protein were significantly reduced, confirming the fact of functional weakness of liver cells, in particular, protein synthesis function.

As it is well known, an acetaminophen intoxication leads to the death of hepatocytes by apoptosis and necrosis. Considering the mechanisms of pharmacological correction of acute hepatic failure with selenolin, it can be assumed that organic selenium improves the reparative processes in damaged hepatocytes, increases the number of ribosomes and polysomes in the affected cells, preventing the further development of pathological disorders, and also increases the synthesis of glutathione, which contributes to an increase in the general potential of the body and lipoperoxides detoxification. Glutathione peroxidase decomposes and detoxifies hydrogen peroxide and other hydroperoxides, protects cell membrane structures and organelles, primarily mitochondria from LPO. In addition, selenium is part of thioredoxin-reductase, which ensures cell resistance to oxidative stress and selenium-dependent glutathione reductase.

Therefore, during the experiment, we assessed the antioxidant activity of selenolin under conditions of acetaminophen intoxication (Table 2).

Table 2. Influence of selenolin on lipid peroxidation parameters in model liver damage with acetaminophen (paracetamol), $\mathrm{M} \pm \mathrm{m}, \mathrm{n}=5$

\begin{tabular}{|c|c|c|c|c|}
\hline \multirow{2}{*}{ Indicators } & \multicolumn{4}{|c|}{ Groups of animals } \\
\cline { 2 - 5 } & Experimental 1 & Experimental 2 & Control & Intact \\
\hline $\mathrm{DC}_{(232)}, \mathrm{AU}$ & $0.74 \pm 0.02$ & $0.67 \pm 0.03^{*}$ & $1.03 \pm 0.01$ & $0.32 \pm 0.01$ \\
\hline $\mathrm{KD}_{(273)}, \mathrm{AU}$ & $0.28 \pm 0.01^{*}$ & $0.25 \pm 0.02$ & $0.46 \pm 0.02$ & $0.14 \pm 0.01$ \\
\hline $\mathrm{MDA}_{(537)}, \mu \mathrm{M} / 1$ & $1.97 \pm 0.14$ & $2.31 \pm 0.09$ & $2.46 \pm 0.11$ & $2.34 \pm 0.16$ \\
\hline
\end{tabular}

Differences are significant $* \mathrm{p}<0.05$ in comparison with the control group of animals

The data in the table show that the course use of selenolin significantly reduced the level of primary lipid peroxidation products in animals of the experimental groups, which follows from a decrease in the content of diene conjugates and ketodienes in comparison with the control group of rats. The discrepancy between the prooxidant and antioxidant resources of hepatocytes due to necrotization of the cell membrane led to an increase in the level of DC in the blood of control animals in 3.22 times relative to intact rats. Whereas in the groups of animals with intoxication, which were injected with a selenium preparation, there was a dynamic decrease in the value of indicators. Thus, in the first experimental group, the level of DC in the blood of rats by the end of the studies decreased in 1.4 times, in the second experimental group it decreased in 1.53 times $(p<0.05)$ relative to the control group.

The concentration of ketodienes in these groups decreased by $39.1 \%(p<0.05)$ and by $56.3 \%$ with their consistent tendency to the level of indicators of healthy animals.

The level of malondialdehyde decreased only in the first experimental group (by $15.8 \%$ relative to healthy rats and by $19.9 \%$ relative to negative control animals) without reliable assessment criteria. It should be noted that malondialdehyde is formed in the body during the degradation of polyunsaturated fats, being a marker of oxidative stress. As shown by the results of the study, in acute liver damage as a result of drug intoxication, the MDA indicator cannot serve as a prognostic criterion for reducing LPO processes in animals.

\section{Conclusion}

Thus, the results of the experiment detrmine that the use of selenolin inhibits and weakens the hepatotoxic effects induced by acetaminophen (paracetamol). The role of selenium is to trap free radicals or to inhibit them, which determines the increase in the antioxidant activity of the body in the processes of lipid peroxidation at the first level of the lipo- 
peroxidation cascade process. Selenolin, enhancing the activity of glutathione peroxidase, protects the secondary metabolization of lipid peroxidation products by neutralizing the already formed hydroperoxides of fatty acids, and preventing their new formation, and also leads to inhibition of the formation of primary toxic products of lipid peroxidation aldehydes, which allows the body to quickly cope with pathological changes in liver cells and in the blood homeostasis system.

\section{References}

1. M. Semenenko, O. Lanets, A. Abramov, E. Kuzminova, I. Zholobova, E3S Web Conf., 210, 06001 (2020)

2. M.P. Semenenko, E.V. Tyapkina, E.V. Kuzminova, A.G. Koschaev, Agricultural biology, 54(4), 777 (2019)

3. E.V. Kuzminova, M.P. Semenenko, S.I. Kononenko, A.A. Abramov, E.P. Dolgov, Asian Journal of Pharmaceutics, 12(4), 1236 (2018)

4. A.N. Mironova, Guidelines for conducting preclinical studies of preparations, 944 (2012)

5. M. Akil, U. Gurbuz, M. Bicer, I. Halifeoglu, A.K. Baltaci, R. Mogulkoc, Bratisl Lek Listy, 116(4), 233 (2015)

6. H. Tapiero, D.M. Townsend, K.D. Tew, Biomed Pharmacother., 57(3-4), 134 (2003)

7. W. Amraoui, N. Adjabi, F. Bououza et al., Toxicol Res., 34(3), 231 (2018)

8. I. Lopez Heras, M. Palomo, Y. Madrid, Anal Bioanal Chem., 400(6), 1717 (2011)

9. R.R. Ramoutar, J.L. Brumaghim, Cell Biochem Biophys., 58(1), 1 (2010)

10. H. Steinbrenner, B. Speckmann, L.O. Klotz, Arch Biochem Biophys., 595, 113 (2016)

11. H. Zhang, P. Limphong, J. Pieper, Q. Liu, C.K. Rodesch, E. Christians, I.J. Benjamin, FASEB J., 26(4), 1442 (2012)

12. N. Metanis, D. Hilvert, Curr Opin Chem Biol., 22, 27 (2014) 